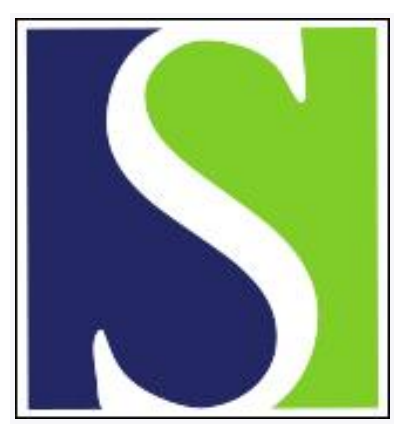

Scand J Work Environ Health 2005;31(3):224-226

https://doi.org/10.5271/sjweh.873

Issue date: Jun 2005

Irritant vocal cord dysfunction at first misdiagnosed as reactive airway dysfunction syndrome

by Galdi E, Perfetti L, Pagella F, Bertino G, Ferrari M, Moscato G

Affiliation: Servizio di Allergologia e Immunologia Clinica, Fondazione Salvatore Maugeri, IRCCS, Località Cravino, 27100 Pavia, Italy. egaldi@fsm.it

The following article refers to this text: 2007;33(2):153-159

Key terms: case report; diagnosis; irritant vocal cord dysfunction; misdiagnosis; occupational asthma; reactive airway dysfunction syndrome

This article in PubMed: www.ncbi.nlm.nih.gov/pubmed/15999575

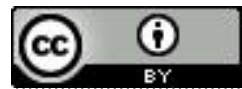




\title{
Irritant vocal cord dysfunction at first misdiagnosed as reactive airway dysfunction syndrome
}

\author{
by Eugenia Galdi, MD, ${ }^{1}$ Luca Perfetti, MD, ${ }^{1}$ Fabio Pagella, MD, ${ }^{2}$ Giulia Bertino, MD, ${ }^{2}$ Massimo Ferrari, \\ $M D,{ }^{3}$ Gianna Moscato, $M D^{1}$
}

Galdi E, Perfetti L, Pagella F, Bertino G, Ferrari M, Moscato G. Irritant vocal cord dysfunction at first misdiagnosed as reactive airway dysfunction syndrome. Scand J Work Environ Health 2005;31(3):224-226.

\begin{abstract}
Objective This report describes a case of vocal cord dysfunction at first misdiagnosed as reactive airway dysfunction syndrome (RADS).

Methods A woman developed recurrent episodes of cough, dyspnea, and wheezing unresponsive to asthma therapy after irritant exposure to glutaraldehyde. Direct laryngoscopy was performed immediately after the induction of symptoms.

Results Laringoscopy showed a paradoxical adduction of the vocal cord on inspiration. Vocal cord dysfunction was diagnosed.

Conclusions A case of vocal cord dysfunction occurred after exposure to glutaraldheyde in a person with a history highly suggestive of RADS. Vocal cord dysfunction should always be considered in the differential diagnosis of patients with acute respiratory symptoms after exposure to irritants and with asthma-like symptoms that fail to respond to conventional asthma therapy.
\end{abstract}

Key terms diagnosis; misdiagnosis; occupational asthma.

Vocal cord dysfunction is a disorder of the larynx characterized by an inappropriate adduction of the anterior two-thirds of the vocal cords during the respiratory cycle, leaving only a posterior "chink" opening resulting in upper airway obstruction (1). It is a poorly understood disease, often misdiagnosed as asthma, and it can coexist with asthma. The patients complain of wheezing, cough, stridor, and dyspnea (2). Symptoms of vocal cord dysfunction often mimic symptoms of bronchial asthma, but they do not improve with the use of corticosteroids and bronchodilators; occasionally intubation or tracheotomy is considered necessary. The diagnosis of vocal cord dysfunction is made by direct visualization of the abnormal movement of the vocal cords (anterior vocal cord adduction with a small opening of the posterior aspect of the cords) (3). Recognition of this condition is essential for a correct diagnosis, to avoid potentially unnecessary therapy, and for appropriate care.
Vocal cord dysfunction has been reported after irritant inhalation exposure $(4,5)$.

In this presentation, we report a case of vocal cord dysfunction that started after irritant exposure to glutaraldehyde, in which, at first, reactive airway dysfunction syndrome (RADS) had been suspected.

\section{Case report}

A 47-year-old nurse was exposed to glutaraldehyde in July 2001 for a time longer than usual while sterilizing devices for transesophageal echocardiography (20-30 minutes instead of 5-10 minutes). Within a few minutes she experienced sore throat, burning eyes, and dry cough. Later she also developed dyspnea and wheezing. She was treated at an emergency room with inhaled salbutamol, inhaled beclomethasone, and intravenous methylprednisolone, with relief of symptoms. The

1 Servizio Autonomo di Allergologia e Immunologia Clinica (Allergy and Clinical Immunology Unit), Fondazione "S Maugeri”, Clinica del Lavoro e della Riabilitazione, IRCCS, Istituto Scientifico di Pavia, Italy.

2 Department of Otorhinolaryngology, University of Pavia, IRCCS Policlinico San Matteo, Italy.

3 Operative Unit of Occupational and Environmental Medicine - Fondazione S Maugeri, Pavia, Italy; and Department of Preventive, Occupational and Community Medicine, University of Pavia, Italy.

Correspondence to: Dr Gianna Moscato, Servizio di Allergologia e Immunologia Clinica, Fondazione Salvatore Maugeri, IRCCS, Località Cravino, 27100 Pavia, Italy. [E-mail: gmoscato@fsm.it] 
following day she experienced acute dyspnea again and returned to the emergency room, where stridor and respiratory distress were observed. The patient was treated at the chest disease department with intravenous aminophylline, intravenous corticosteroids, inhaled corticosteroids, and inhaled salbutamol. Bronchial asthma due to the inhalation of glutaraldehyde was suspected, and the patient was put on regular therapy with inhaled formoterol $(24 \mu \mathrm{g} /$ day $)$, inhaled fluticasone $(1000 \mu \mathrm{g} /$ day), and inhaled salbutamol as needed. A methacholine challenge was scheduled. The patient was unable to perform the challenge due to acute dyspnea and stridor after basal spirometry. Spirometry was normal. Neck nuclear magnetic resonance and chest computed tomography produced normal results. One month later, the patient returned to work and was asymptomatic for the following 2 months. Again methacholine challenge was not performed due to stridor and acute respiratory distress after basal spirometry. Symptoms reappeared despite regular antiasthma therapy, and on one occasion she was treated at the emergency room with intravenous methylprednisolone, intravenous atropine, and intravenous aminophylline. A chest physician prescribed a short course of oral prednisone. Because of the persistence of the symptoms and the suspected relation with work exposure, the patient consulted an occupational physician, who suspected RADS and referred the patient to our allergy unit. When we saw the patient in January 2002 , she was on therapy with fluticasone $(1000 \mu \mathrm{g} / \mathrm{day})$ and formoterol ( $24 \mu \mathrm{g} /$ day $)$, unsuccessfully.

The patient's personal and familial histories were negative for atopy. Her chest $\mathrm{X}$ ray was normal. Her skin prick test and test for specific immunoglobulin (Ig) $\mathrm{E}$ for common aeroallergens were negative, except IgE for cat dander. The total IgE was $66.1 \mathrm{kU} / \mathrm{l}$. Spirometry showed a vital capacity of 3.611 (118\% of the predicted value) and a forced expiratory volume in 1 second $\left(\mathrm{FEV}_{1}\right)$ of 3.051 ( $116 \%$ of the predicted value). Immediately after the spirometry, the patient experienced cough, acute dyspnea, and stridor, which disappeared spontaneously within about 1 minute. Vocal cord dysfunction was suspected.

The patient was submitted to fiberoptic laryngoscopy. No lesions were found on the vocal cords, and no abnormal movements were observed on quiet breathing. After appropriate self-induction maneuvers (6), a paradoxical adduction of the vocal cords on inspiration producing the characteristic posterior "chink" opening was observed (figure 1). Symptoms were relieved by panting (7).

A psychological consultation revealed anxiety and a reduced mood level resulting in a diagnosis of reactive depression related to a recent familial stressor.

On the basis of the clinical history, symptoms, and the typical laryngoscopic finding of adduction of the anterior two-thirds of the vocal cords on inspiration, the diagnosis of vocal cord dysfunction was made.

\section{Discussion}

We report a case of vocal cord dysfunction after exposure to glutaraldheyde in a nurse with a history suggestive of reactive airway dysfunction syndrome (RADS).

RADS is a type of occupational asthma that can occur after single or multiple exposures to nonspecific irritants at high concentrations, and it is characterized by the absence of a latency period (8). In this case, the history was misleading. The accidental exposure to glutaraldehyde and symptoms mimicking asthma suggested RADS. RADS is characterized by persistent bronchial hyperreactivity, but, in this case, a methacholine challenge could not be performed due to acute dyspnea after basal spirometry.

Two types of vocal cord dysfunction have been described, irritant vocal cord dysfunction and nonirritant vocal cord dysfunction (5). Our case met the criteria for irritant vocal cord dysfunction (absence of preceding vocal cord dysfunction or laryngeal disease, onset of symptoms after a single specific exposure to an irritant vapor, onset of symptoms within 24 hours after exposure, symptoms of wheezing, stridor, dyspnea, cough, abnormal direct laryngoscopy for vocal cord dysfunction with a provocative study, exclusion of other vocal cord diseases) (5).

Our case confirms that an acute onset of respiratory symptoms after exposure to irritants should raise a suspicion of vocal cord dysfunction, as well as that of

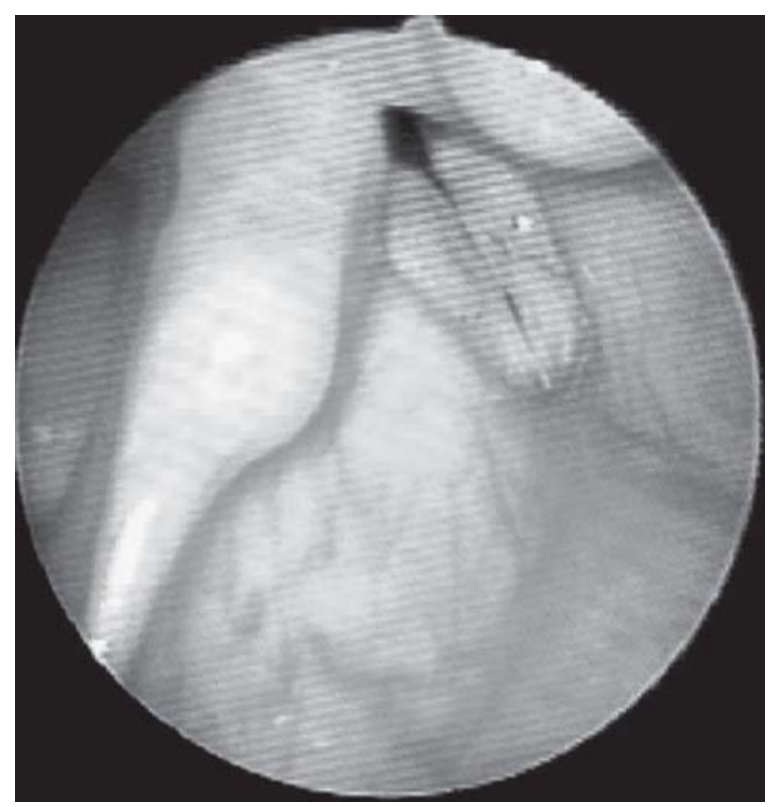

Figure 1. Endoscopic view showing the posterior glottal chink. 
RADS, to avoid inappropriate treatment and to provide appropriate care. Vocal cord dysfunction should primarily be suspected for patients who fail to respond to asthma therapy. The impossibility to perform a methacholine challenge did not allow us to exclude coexisting RADS with objective methods, but features of the symptoms and the failure to control the symptoms with conventional asthma therapy make RADS unlikely.

In a retrospective survey, Newman et al (1) showed that patients with vocal cord dysfunction are predominantly women, health care workers, and persons with mental disorders. Mental disorders associated with vocal cord dysfunction include depressive and anxiety disorders, personality disorders such as obsessive-compulsive disorder and borderline personality disorder. It has been hypothesized that vocal cord dysfunction in these patients may be a conversion reaction (9). Our report met these characteristics. The patient was a female. She had been working as a nurse for several years. And she had an anxiety and depressive disorder. Although the relationship between vocal cord dysfunction and psychopathology is still unclear (5), a psychological evaluation for patients with vocal cord dysfunction is important to reveal psychological disorders in order to provide appropriate management. There are several treatment options for acute management, for example, panting, coughing and breathing against pressure may terminate an acute attack, and diverting the patient's attention from inspiration to expiration by asking the patient to make a soft "s" sound while exhaling may also be helpful. Severe dyspnea can be effectively treated with a mixture of helium and oxygen. The first step of long-term therapy is to inform the patient of the functional nature of the disease. Speech therapy and psychological counseling are then the two main treatment modalities (7).

In conclusion, vocal cord dysfunction should always be considered in the differential diagnosis of patients with acute respiratory symptoms after exposure to irritants and with asthma-like symptoms which fail to respond to conventional asthma therapy. A correct diagnosis is basic for the initiation of appropriate medical intervention or a rehabilitation program.

\section{References}

1. Newman KB, Mason UG, Shmaling KB. Clinical features of vocal cord dysfunction. Am J Respir Crit Care Med 1995; 152:1382-6.

2. Newman KB, Dubester SN. Vocal cord dysfunction: masquerade of asthma. Semin Respir Crit Care Med 1994;15:162-7.

3. Christopher KL, Wood RP, Eckert RC, Blager F, Raney R, Souhrada J. Vocal-cord dysfunction presenting as asthma. N Eng J Med 1983;308:1566-70.

4. Mason UG, Fennelly K, Newman K, Wood R, Blager F. Vocal cord dysfunction from irritant exposure. Clin Res Allergy Hypersensitivity 1994;438:1588.

5. Perkner JJ, Fennelly KP, Balkissoon R, Bartelson BB, Ruttenber AJ, Wood RP 2nd, et al. Irritant-associated vocal cord dysfunction. J Occup Environ Med 1998;40:136-43.

6. Wood RP 2nd, Milgrom H. Vocal cord dysfunction. J Allergy Clin Immunol 1996;98:481-5.

7. Bahrainwala AH, Simon MR. Wheezing and vocal cord dysfunction mimicking asthma. Curr Opin Pulm Med 2001;7:813.

8. Malo J-L, Chan-Yeung M. Occupational asthma. J Allergy Clin Immunol 2001;108:317-28.

9. Christopher KL, Wood RP, Eckert C, Blager FB, Raney RA, Souhrada KL. Vocal cord dysfunction presenting as asthma. N Engl J Med 1983;308:1566-70.

Received for publication: 21 June 2004 